\title{
Neurological Disorders in Children / the Sheikh's Syndrome
}

\author{
Prof. Dr. Shahid Hussain Sheikh, $M B B S, P h D$. \\ Chairman: NIDS Treatment \& Research Center \\ Department of Neurology \& Neuro Virology \\ United Christian Hospital, \\ Gulberg III, Lahore, Pakistan
}

\begin{abstract}
The label of "Cerebral Palsy" has been used frivolously due to the lack of knowledge of the Brain's pathology. Since 2004, a number of researchers have stumbled upon a new paradigm in the field of Neurology ${ }^{I}$ that now opens up a new door of hope to better the quality of life of the suffering infants, children and adolescence worldwide.

Now, many children with CP are known to be suffering from the Neuronal Sub-Nucleus Neuro viral RNA proliferation that begins as early as the inception of a new baby. The genetically transforming diseases now take a back seat to this new revelation, where, the Neuro Viral RNA's being part of the chromosomal DNA of the sperm or the Ovum also ready to proliferate in the developmental stage of the Neuro Disk of the fetus in the first trimester.

Therefore, the brain, spinal cord and all nerves may have the greater architectural influence of the Neuro Viral RNA Proliferation. So much so that, this influence may be carried out in to the developing functions of the organelles, cells, membranes, tissues, organs, systems.

All cells, membranes, tissues, organs and systems are constructed with the Sensory Nerves and Motor Nerves, therefore, connected and controlled by the peripheral and central nervous system.

On the other hand, maternal Immunity interferes in the defense, so long to protect the developmental anomalies, equal to the potency of the hosting mother's immunity. If the virion / RNA proliferation is faster than the production and potency of the immune cells, of course, the battle is going to be lost.

Baby is going to be presenting the pregnancy related challenges, and if born, may have some serious developmental challenges.

Any Neuronal anomaly among the billions of developing neurons will translate in to what we know today as the Cerebral Palsy, Late Milestone, Autism, Hydrocephaly etc.

It is now discovered that Neuro Viral RNA proliferation does take place due to the auto immune disorder, in particular, the Neuro Immune Dysfunction Syndrome now further defined as

"Sheikh's Syndrome" (A Multi Neuronal Sub-Nucleus Neuro Viral RNA Proliferation).
\end{abstract}

\section{Introduction:}

Immunity's incapability of matching up to defend the nucleus of the neuron enables the temporary cessation of the homeostasis of that controlling neuron or a group of neurons. Therefore, that particular neuron or a cluster of neurons will not participate in the required function to their fullest capacity. Therefore, the state of homeostasis is disrupted. The whole hierarchy of the function from the cell to the system level is negatively affected.

$\mathrm{CP}$ is no different than this. However, it depends upon the level of disruption that has been commenced by this foreign body in the development stage.

Miscarriages and other pregnancy related complications are presented as the prelude to the fetal risk of the incomplete development. Neo-natal risks need to be further researched in the light of this new dimension of Neuro Virology.

\section{Materials \& Methods:}

Our research center has been actively studying the children of 1 year to 19 years of age with a variety of "CP" and has been able to help them reclaim a normal quality of life including but not limited to the following:

1. Regaining/ Start of the speech

2. Correctness of the upper and Lower Limbs (No Surgery)

3. Hydrocephaly (No Shunt)

4. Adolescent Neurological Disorders

5. Correctness of Cross Eyes

6. Standing \& Walking 
Selective antiviral therapy has been infused via I.V. in an O.D. and B.I.D. dose according to the body weight.

Supportive meds were also used to keep the homeostasis, i.e. Mecobalamin, Prednisone/ Dexamethasone along with pain killers and antihistamine. All patients responded well to this strategy with out any event.

\section{Results:}

Most of all patients seen improved quality of life and rid of physical handicap in speech; motor function improvement and limited self sufficiency. Further long term studies need to be conducted to further evaluate this new paradigm.

\section{Discussion:}

United States: Approximately $65 \%$ of the population is seropositive for HSV-1 by the fourth decade of life. Approximately $25 \%$ of the population is seropositive for HSV-2 by the fourth decade of life, with women being infected more frequently than men.

United Kingdom: By age 15 around $25 \%$ of population, by age 30 around $50 \%$ inflicted by herpes ${ }^{2}$.

Europe: The seroepidemiology of herpes simplex virus type 1 and 2 in: Large intercountry and intracountry differences in HSV-1 and HSV-2 seroprevalence were observed. Age standardized HSV-1 seroprevalence ranged from 52\% in Finland, to 57\% in the Netherlands, 67\% in Belgium, $81 \%$ in Czech Republic, and $84 \%$ in Bulgaria $^{3}$.

\section{Conclusion:}

We continue to address this untapped paradigm that has opened up the door for the children yet suffering from these solvable temporary anomalies. We believe that the Neurons are always developed to their fullest capacity, purity and normalcy; it is the Neuro viral disease that influences the normal functioning.

Therefore, by helping the patient lower the viral influence and help the resident immunity, we can make a miraculous difference in one's life.

It is not only the disease for the children; the same can appear in anytime of one's life.

As a matter of fact, now many leading scientists of the world begin to point towards the Neuro Virological aspects of the primary diagnosis of the major diseases that are claiming lives, i.e. Blood Pressure, Stroke and Bells Palsy etc.

At the opening ceremony of the Nobel Prize 2011, a scientist started his address by saying: "Excellencies, fellow scientists, ladies and gentlemen, tonight we are going to exchange a lot of viruses". Long behold, it is so true, in every society viruses are the most prevalent and considered widely ignored. And we wonder about the etiologies.

Pre-natal vitamins therapy now needs a serious $2^{\text {nd }}$ look, whether or not it is sufficient to protect the fetal development from the viral RNA integration. The time has come now where, before planning a pregnancy a Neuro Virologist is consulted to rectify any Neuro invasive viral pathology if present in the expecting couples and screen throughout the pregnancy for any possible neurological anomalies.

Of course, after delivery of the baby, correcting the Neuro Viral challenges warrant.

NIDS Treatment \& Research Center Trust at the Department of Neurology and Neuro Virology,

United Christian Hospital is the pioneer to introduce this new paradigm for the best baby health.

Acknowledgements:

I am personally very much thankful to the following peers for the critical review of my work.

1. Dr. Emmanuel Bhatti, Medical Director United Christian Hospital, Lahore, Pakistan.

2. Dr. Farhat Zarin, Chief Obstetrician \& Gynecologist, Department of Labor \& Delivery, United Christian Hospital, Lahore, Pakistan.

3. Dr. Mujahid Hussain, Sexologist, NIDS Treatment \& Research Center, Lahore, Pakistan.

\footnotetext{
[1] Plomp et al, AJP June 2002, Vol. 160, No. 6

[2] www.herpes.org.uk

[3] www.who.org
}

\section{References:}

